\title{
Low power analog frontend for ISFET sensor readout
}

\author{
Jun-Rui Zhang ${ }^{1}$, F. Bellando ${ }^{1}$, E. A. Garcia Cordero ${ }^{1}$, M. Mazza $^{2}$, M. Fernandez-Bolanos ${ }^{1}$ and A. M. \\ lonescu ${ }^{1}$ \\ ${ }^{1}$ Nanolab, École Polytechnique Fédéral de Lausanne, Lausanne, Switzerland, \\ ${ }^{2}$ University of Applied Science Western Switzerland, Fribourg, Swizerland \\ Junrui.zhang@epfl.ch
}

\begin{abstract}
:
A low power analog frontend (AFE) for Ion-Sensitive Field effect Transistor sensor readout is presented. The AFE is demonstrated with off-the-shelf components. It includes a biasing circuit to bias an ISFET sensor, a noise rejecting current readout circuit, and a sigma-delta analog to digital converter. The digital output can be interpreted by a simple counter to acquire the ion-concentration in a drop of liquid on top of the calibrated ISFET sensor. A PH sensing experiment is performed to validate the AFE. Total power consumption is less than $40 \mu \mathrm{W}$ with $1.8 \mathrm{~V}$ supply.
\end{abstract}

Key words: Analog frontend; Current mode sensor; Phase sensitive detection; Current rectifier; Current multiplier.

\section{Introduction}

ISFET (Ion-Sensitive Field Effect Transistor) sensor is a type of sensors that exploits the ionconcentration influence on its $I-V$ curve. An ISFET works like a MOSFET whose gate metal is removed (Fig. 1a). The gate oxide is covered by a sensitive layer and immersed in the liquidunder-test for trapping certain targets (ions, biomarkers, etc.). As shown in Fig. $1 b$, when the reference electrode (immersed in the liquidunder-test) is biased properly, the surface potential $\Psi_{S}$ of the ISFET's channel will be linearly related to $V_{\text {ref. }}$. At the same time, $\Psi_{S}$ can be modified by chemical events when the target is trapped on-site. The amount of modification depends on the concentration of the target. Thus, bio/chemical events can be translated into electrical signals by ISFET sensors.

This paper presents an analog frontend (AFE) sensor readout circuit (Fig.2) that biases the ISFET sensor and reads the corresponding sensor current into digital stream. Adopting phase sensitive detection (PSD) technique, [1] the readout circuit is able to move the low frequency noise (accompanied with the sensor current) to higher frequency band. A low pass filter followed by a $1^{\text {st }}$-order sigma-delta $A D C$ is used to convert the low-noise signal into digital stream.

The readout circuit is demonstrated in PCB with off-the-shelf components, consumes less than $40 \mu \mathrm{W}$ with $1.8 \mathrm{~V}$ supply.

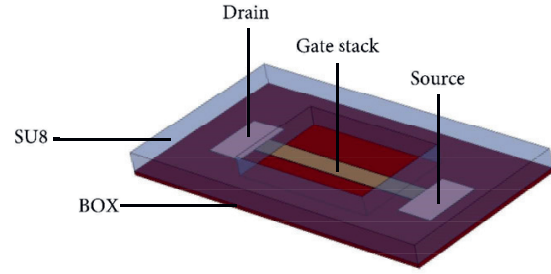

a

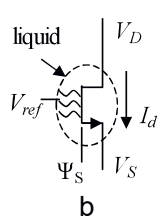

Fig. 1 Ion-Sensitive Field Effect Transistor a Layout representation of the proposed ISFET $b$ A sketch of the ISFET sensor. $V_{\text {ref: }}$ reference electrode voltage, $\boldsymbol{\psi}_{\mathrm{s}}$ : surface potential, $V_{D / S}$ : drain/source bias voltage, $I_{d}$ : drain current. $\psi_{S}$ is linearly proportional to $V_{\text {ref }}$ and monotonically varying with the amount of targets trapped onsite. Id varies monotonically with $\psi_{S}$.

\section{Principle of operation and circuit implementation}

The proposed AFE is shown in Fig. 2. $V_{D S}$ is an AC signal of frequency $f_{o}=77 \mathrm{~Hz}$ that excites the device under test (DUT), in order to modulate the sensor information into a sensor current $I_{\text {SENSE }}$ of carrier frequency $f_{o}$. The DUT adopted here is an ISFET sensor, whose gate oxide is sensitive to $\mathrm{H}^{+}$concentration. Due to the nonlinear $I_{d}-V_{\text {ref }}$ behavior of ISFETs, the AFE needs to apply a square wave across $V_{D S}$ when 
sensing. For ease of interpreting the circuit principle, $V_{D S}$ is drawn here as a sinusoid. Since the DUT has symmetric drain and source connections, the sensor current $I_{S E N S}$ is also a square wave, symmetrical with respect to 0 ampere. The square wave current has two states. In state $S 1$, when $I_{S E N S}$ is negative: $V_{r}$ is high, ISENS is conducted through $\mathrm{M} 1$, and mirrored by CM1 to $I_{O}$; in state $S 2$, when $I_{S E N S}$ is positive: $V_{r}$ is low, $I_{S E N S}$ is conducted through $\mathrm{M} 2$, and mirrored by $\mathrm{CM} 2$ to $I_{O}$. Adding the two states together, makes $I_{O}$ the rectified version of the sensor current.

$$
I_{O}=I_{\text {SENS }} \times V_{r}
$$

Thus, through the bidirectional negative feedback current conveyer formed by the opamp and transistors M1-M2, the input current $I_{\text {SENS }}$ is mixed with $V_{r}$. Interestingly, $V_{r}$ is a square wave voltage whose phase is locked with the excitation signal $V_{D S}$ via the same feedback loop.

With Eq. (1), the sensor information carried in the square wave current is demodulated back to $D C$ frequency, and the low frequency noise is shifted to a frequency band centering at $f_{0}$. Then $I_{O}$ is passed through an LPF to remove the noise at high frequency, and obtain a DC current $I_{O F}$ that varies monotonically with the amplitude of $I_{\text {SENS. A sigma delta ADC is used }}$ to readout the filtered current. Since the input is a DC current, a simple counter can be used instead of a complicated decimation filter. The counter counts the number of rising edges at the output $\left(D_{O U T}\right)$, which is proportional to the amplitude of the input current $\left(I_{O F}\right)$.

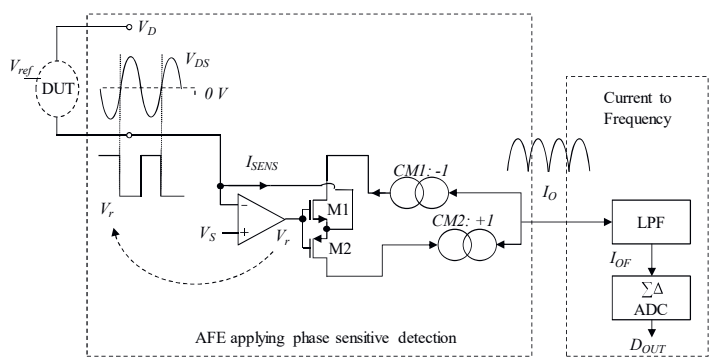

Fig. 2 Block diagram of the proposed AFE. $V_{r}$ (phase locked to the excitation signal $V_{D S}$ ) is generated out of the biasing circuit. $V_{D S}$ is drawn here as a sinusoid. The system actually applies square wave for easy implementation.

\section{Measurement Results}

An n-channel ISFET on ultra-thin SOI with $\mathrm{HfO} 2$ as its gate oxide has been applied for $\mathrm{PH}$ sensing. [2] The sensor was biased with voltage while sampling its drain current. Different $\mathrm{PH}$ buffers were changed consecutively from $\mathrm{PH}=3$ to $\mathrm{PH}=9$, and back from 9 to 3 during the measurement. The measurements were done both with the proposed AFE and a precision semiconductor analyzer (Agilent 4156A), in order to validate the performance of the readout AFE. (a) With Agilent 4156A, the ISFET was biased at $\mathrm{V}_{\text {ref }}=1.3 \mathrm{~V}, \mathrm{~V}_{\mathrm{S}}=800 \mathrm{mV}, \mathrm{V}_{\mathrm{D}}=1.1 \mathrm{~V}$. (b) With the AFE, the ISFET was biased at $V_{\text {ref }}=1.3$ $\mathrm{V}, \mathrm{V}_{\mathrm{L}}=800 \mathrm{mV}, \mathrm{V}_{\mathrm{H}}=1.1 \mathrm{~V} \mathrm{~V}_{\mathrm{L}}$ and $\mathrm{V}_{\mathrm{H}}$ voltages applied on the Drain and source alternatively, forming a square wave $V_{D S}$ at a frequency of 77 $\mathrm{Hz}$ ). The measurement results are shown in Fig.3. The digital output from the AFE was counted for the number of rising edges, and proves to be reasonably coherent with lab instrument measurements (Fig. 3).

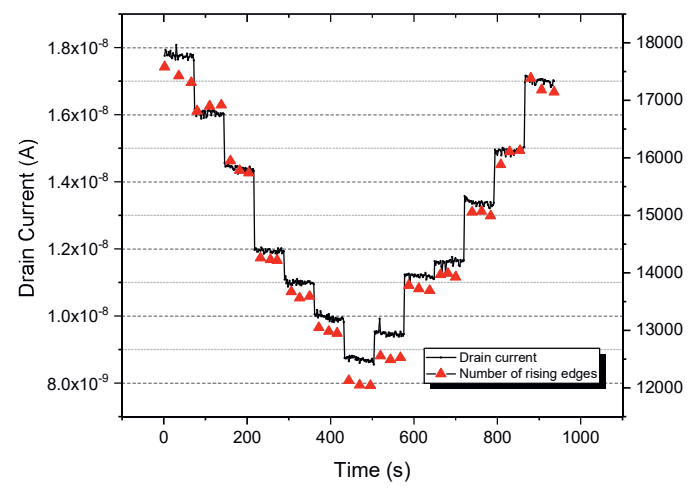

Fig. $3 \mathrm{PH}$ sensing performed to verify the performance of the readout AFE. Left axis: measurements performed by Agilent 4156A; Right axis: number of rising edges from the presented AFE

\section{Conclusions}

An ISFET sensor readout AFE is demonstrated. It modulates the sensor information with a square wave signal by applying alternating bias to the sensor; adopts a novel current mode structure in the lock-in amplifier. A PH sensing experiment validates that the readout AFE is able to work with ISFET sensors by biasing them in amperometric mode, and provide good current readout results. The system operates at $1.8 \mathrm{~V}$ supply, consumes less than $40 \mu \mathrm{W}$, and the simple structure makes it suitable to be integrated in a single chip.

\section{References}

[1] A. De Marcellis, et, al, "One-Decade Frequency Range, In-Phase Auto-Aligned $1.8 \mathrm{~V} 2 \mathrm{~mW}$ Fully Analog CMOS Integrated Lock-In Amplifier for Small/Noisy Signal Detection," in IEEE Sensors Journal, vol. 16, no. 14, pp. 5690-5701, July15, 2016.

[2] F. Bellando, et, al, "Lab on $\operatorname{skin}^{\mathrm{TM}}$ : 3D monolithically integrated zero-energy micro/nanofludics and FD SOI ion sensitive FETs for wearable multi-sensing sweat applications," 2017 IEEE International Electron Devices Meeting (IEDM), San Francisco, CA, 2017, pp. 18.1.1-18.1.4. 